\title{
Expanding the phenotype of de novo SLC25A4- linked mitochondrial disease to include mild myopathy
}

Martin S. King, PhD, * Kyle Thompson, PhD,* Sila Hopton, BSc, Langping He, PhD, Edmund R.S. Kunji, PhD, Robert W. Taylor, PhD, FRCPath, and Xilma R. Ortiz-Gonzalez, MD, PhD

Neurol Genet 2018;4:e256. doi:10.1212/NXG.0000000000000256

\section{Abstract}

\section{Objective}

To determine the disease relevance of a novel de novo dominant variant in the SLC25A4 gene, encoding the muscle mitochondrial adenosine diphosphate (ADP)/adenosine triphosphate (ATP) carrier, identified in a child presenting with a previously unreported phenotype of mild childhood-onset myopathy.

\section{Methods}

Immunohistochemical and western blot analysis of the patient's muscle tissue were used to assay for the evidence of mitochondrial myopathy and for complex I-V protein levels. To determine the effect of a putative pathogenic p.Lys33Gln variant on ADP/ATP transport, the mutant protein was expressed in Lactococcus lactis and its transport activity was assessed with fused membrane vesicles.

\section{Results}

Our data demonstrate that the heterozygous c.97A $>\mathrm{T}$ (p.Lys33Gln) SLC25A4 variant is associated with classic muscle biopsy findings of mitochondrial myopathy (cytochrome $c$ oxidase $[\mathrm{COX}]$-deficient and ragged blue fibers), significantly impaired ADP/ATP transport in Lactococcus lactis and decreased complex I, III, and IV protein levels in patient's skeletal muscle. Nonetheless, the expression levels of the total ADP/ATP carrier (AAC) content in the muscle biopsy was largely unaffected.

\section{Conclusions}

This report further expands the clinical phenotype of de novo dominant SLC25A4 mutations to a childhood-onset, mild skeletal myopathy, without evidence of previously reported clinical features associated with SLC25A4-associated disease, such as cardiomyopathy, encephalopathy or ophthalmoplegia. The most likely reason for the milder disease phenotype is that the overall AAC expression levels were not affected, meaning that expression of the wild-type allele and other isoforms may in part have compensated for the impaired mutant variant.

\author{
Correspondence \\ Dr. Ortiz-Gonzalez \\ ortizgonzalez@email.chop.edu
}




\section{Glossary:}

AAC $=$ ADP $/$ ATP carrier ADP $=$ adenosine diphosphate ATP $=$ adenosine triphosphate COX $=$ cytochrome $c$ oxidase; CHOP = Children's Hospital of Philadelphia; $\mathbf{C K}=$ creatine kinase; $\mathbf{m t D N A}=$ mitochondrial DNA; OXPHOS = oxidative phosphorylation; PEO = progressive external ophthalmoplegia.

SLC25A4 (ANT1 and AAC1) gene mutations cause an intriguing spectrum of human disease, with dominant mutations first reported in adults with progressive external ophthalmoplegia (PEO), ${ }^{1}$ whereas recessive loss-of-function mutations cause cardiomyopathy and skeletal myopathy., ${ }^{2,3}$ Furthermore, SLC25A4 de novo dominant mutations can present in neonates with lactic acidosis, severe hypotonia, and respiratory failure. ${ }^{4}$ Here, we report a patient who presented at age 2 years with mild weakness and hypotonia and was found to have a novel de novo heterozygous SLC25A4 variant (c.97A>T;p.Lys33Gln).

The SLC25A4 gene encodes the mitochondrial AAC1, which imports $\mathrm{ADP}$ into the mitochondrion and exports ATP. ${ }^{5}$ Humans have 4 AAC isoforms, with AAC1 being specific to the heart, skeletal muscle, and brain. ${ }^{6}$ Previous functional studies correlating the rate of $\mathrm{ADP} / \mathrm{ATP}$ exchange with phenotype severity have only partially solved the puzzle. In dominant-acting mutations, the rate of transport does seem to correlate with clinical severity, with mutations associated with $\mathrm{PEO}$ having higher residual $\mathrm{ADP} / \mathrm{ATP}$ transport rates compared with de novo mutations associated with severe neonatal disease. $^{4}$ However, loss-of-function recessive mutations present later than de novo cases with a predominant cardiac phenotype, despite in vitro functional studies showing essentially no measurable ADP/ATP exchange ${ }^{4}$

Here, we report that the de novo dominant variant p.Lys33Gln in SLC25A4 is clinically associated with mild myopathy despite significant mitochondrial pathology in muscle biopsy and functional data showing abolished ADP/ ATP exchange in vitro. This case expands the known phenotype of SLC25A4 disease to include childhood-onset mild skeletal myopathy without evidence of cardiac, brain, or extraocular muscle involvement.

\section{Methods}

\section{Case presentation}

A 2-year old girl presented to the neurology clinic at the Children's Hospital of Philadelphia (CHOP) for hypotonia and mild gross motor delays. Neurologic examination at presentation was only remarkable for hypotonia and a 1handed Gower maneuver, suggestive of mild weakness. Family history was unremarkable. Laboratory workup found elevated creatine kinase (CK) (616 U/L, normal 60-305) and lactic acidosis (3.76 mM, normal 0.8-2.0) levels; therefore, muscle biopsy and subsequent genetic testing were pursued. Clinical testing for nuclear mitochondrial disease genes and full mitochondrial DNA (mtDNA) sequencing (in blood and muscle) were obtained via the next-generation sequencing panel.

\section{Standard protocol approvals, registrations, and patient consents}

Informed consent was obtained from the child's parents to enroll in a human subject's research protocol approved by the Institutional Review Board at CHOP.

\section{Human muscle immunohistochemistry and analysis}

Standard histologic and histochemical analyses were performed on $10-\mu \mathrm{m}$ transversely oriented muscle cryosections. Quadruple immunofluorescence analysis of NDUFB8 (complex I) and COXI (complex IV) ${ }^{7}$ and western blot analysis $^{3}$ were performed as previously reported. mtDNA copy number assessment in muscle was undertaken as described. ${ }^{8}$

\section{Functional studies of p.Lys33GIn variant in Lactococcus lactis}

The SLC25A4 gene was cloned into the L. lactis expression vector pNZ8048 by established procedures, ${ }^{9}$ and the p.Lys33Gln variant was introduced and confirmed by sequencing. Growth of L. lactis, membrane isolation, vesicle preparation, transport assays, and western blot analysis were performed as reported. 4

\section{Results}

\section{Patient results}

Histopathologic assessment of skeletal muscle from the patient demonstrated a mosaic pattern of cytochrome c oxidase (COX) deficiency (figure 1A). Quadruple immunofluorescence analysis confirmed a mitochondrial defect involving both complexes I and IV with some fibers exhibiting normal protein expression (figure 1B). MtDNA copy number was decreased to approximately $40 \%$ in patient muscle compared with age-matched controls. Western blot analysis showed a slight decrease in AAC protein levels, associated with more markedly decreased steady-state protein levels of components of respiratory complexes (CI, CIII, and CIV) in patient skeletal muscle (figure 1C).

Genetic testing revealed a heterozygous SLC25A4 variant (c.97A >C, p.Lys33Gln) that was confirmed to have arisen de novo following parental testing. Serial cardiac evaluations including ECG, echocardiogram, and Holter monitoring were unremarkable from diagnosis at age 2 years to current age of 8 

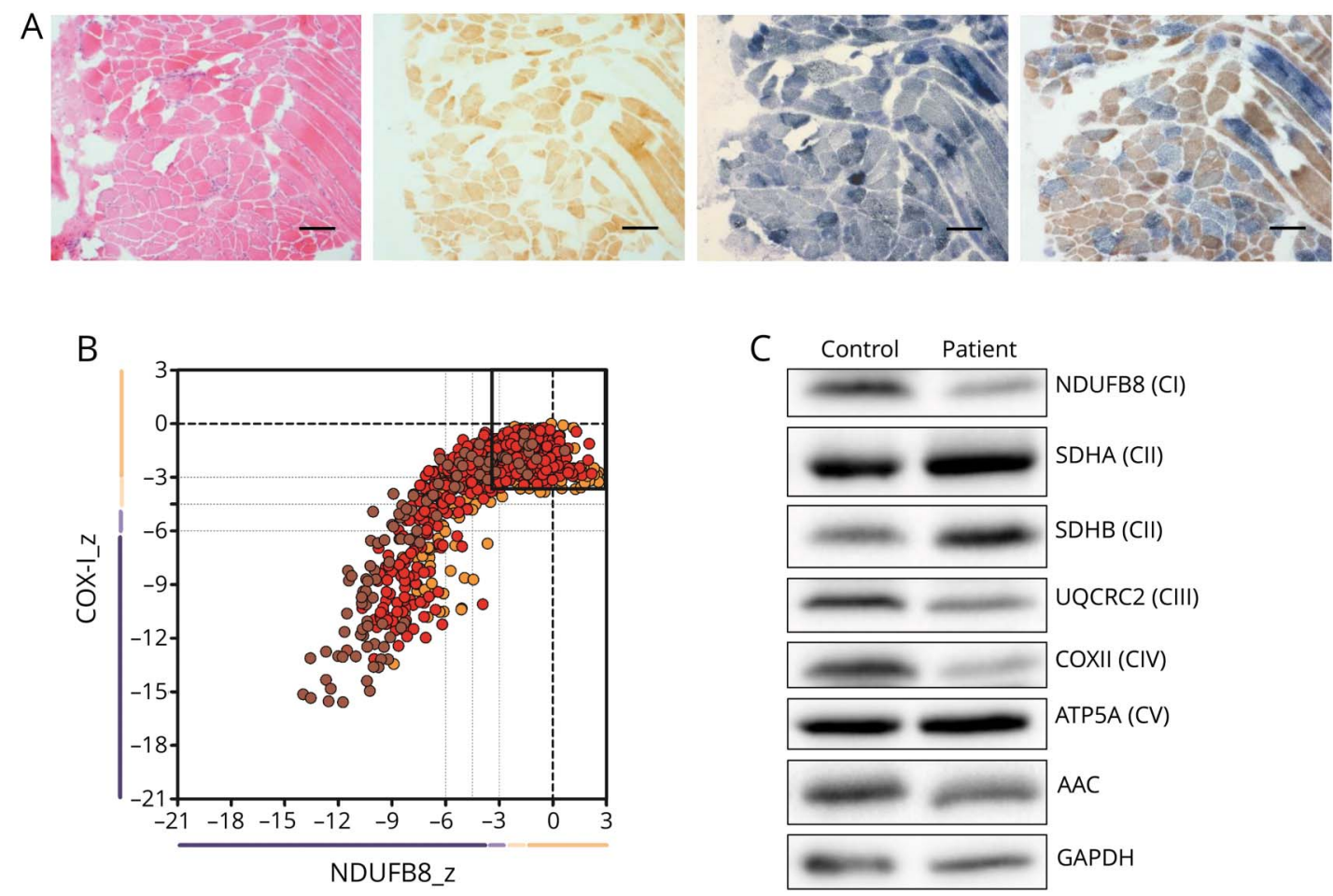

(A) Histopathologic analysis of patient skeletal muscle sections showing hematoxylin and eosin (H\&E) staining (far left), COX histochemistry (middle left), SDH histochemistry (middle right), and sequential COX-SDH histochemistry (far right). Scale bar $=100 \mu \mathrm{m}$. (B) Respiratory chain profile following quadruple oxidative phosphorylation immunofluorescence analysis of cryosectioned muscle from the index case, confirming the presence of fibers lacking complex I (NDUFB8) and complex IV (COXI) protein. Each dot represents the measurement from an individual muscle fiber, color coded according to its mitochondrial mass (blue-low, normal-beige, high-orange, and very high-red). Gray dashed lines indicate SD limits for the classification of fibers. Lines next to $x$ - and $y$-axes represent the levels (SDs from the average of control fibers after normalization to porin/VDAC1 levels; Zz= Z-score, see Methods section of Rocha et al. 2015 for full description of statistics ${ }^{7}$ ) of NDUFB8 and COX1, respectively (beige $=$ normal [>-3], light beige $=$ intermediate positive [ -3 to- -4.5$]$, light purple $=$ intermediate negative $[-4.5$ to -6$]$, and purple $=$ deficient $[<-6]$. Bold dotted lines indicate the mean expression level observed in respiratory normal fibers. (C) Western blot analysis of AAC and OXPHOS complex subunits on control and patient skeletal muscle samples. AAC = ADP/ATP carrier; ADP = adenosine diphosphate; ATP = adenosine triphosphate; $\mathrm{COX}=$ cytochrome $\mathrm{c}$ oxidase; GADPH = Glyceraldehyde 3-phosphate dehydrogenase; OXPHOS = oxidative phosphorylation; SDH = succinate dehydrogenase.

years. Neurologic evaluations remain stable, only remarkable for mild proximal weakness, hyperCKemia, and lactic acidosis, with normal extraocular movements and no cognitive abnormalities.

\section{Assessment of SLC25A4 p.Lys33GIn variant function}

The transport mechanism of SLC25A4 involves the disruption and formation of the matrix and cytoplasmic salt bridge network in an alternative way (figure $2 \mathrm{~A}) .^{5}$ Residue Lys33 in SLC25A4, which is conserved among AAC from fungi, plants, and metazoans (figure 2B), forms a salt bridge with the conserved Asp232 in the matrix network (figure 2, C and D). The p.Lys33Gln mutation would eliminate this interaction, as glutamine is a neutral amino acid residue and too short to form a hydrogen bond (figure $2 \mathrm{E}$ ). Below the salt bridge is Gln 37 that forms a highly conserved glutamine brace ${ }^{10}$ (figure 2, B-D), which would also be disrupted by the mutation (figure 2E).

We introduced the p.Lys33Gln mutation into the human SLC25A4 sequence and expressed it L. lactis membranes. The uptake of radio-labeled ADP in exchange for loaded ADP was measured for an empty vector control (figure 3A), wild-type SLC25A4 (figure 3B), and p.Lys33Gln, using the specific inhibitor carboxyatractyloside as control (figure 3C). The mutant protein is expressed to approximately the same levels as wildtype in lactococcal membranes, suggesting that the mutation does not affect biogenesis, protein folding, or targeting to the membrane in this expression system (figure 3D). The p.Lys33Gln mutant was not able to transport ADP (figure 3E).

\section{Discussion}

Previously, we have functionally characterized the effect of 9 pathogenic variants in human SLC25A4 using L. lactis, determining residual transport activity compared with wild-type protein. ${ }^{4}$ Broadly speaking, residual transport activities of the mutants segregate with the associated clinical phenotype; mutations associated with adult-onset dominant PEO display higher residual transport activities $(24 \%-56 \%)$ than mutations associated with recessive disease, which were effectively nonfunctional. ${ }^{4}$ There was also a correlation between the severity of the clinical phenotype and residual transport activity of previously documented dominant mutations, with 
Figure 2 The p.Lys33GIn mutation eliminates a conserved salt bridge interaction of the matrix network

A

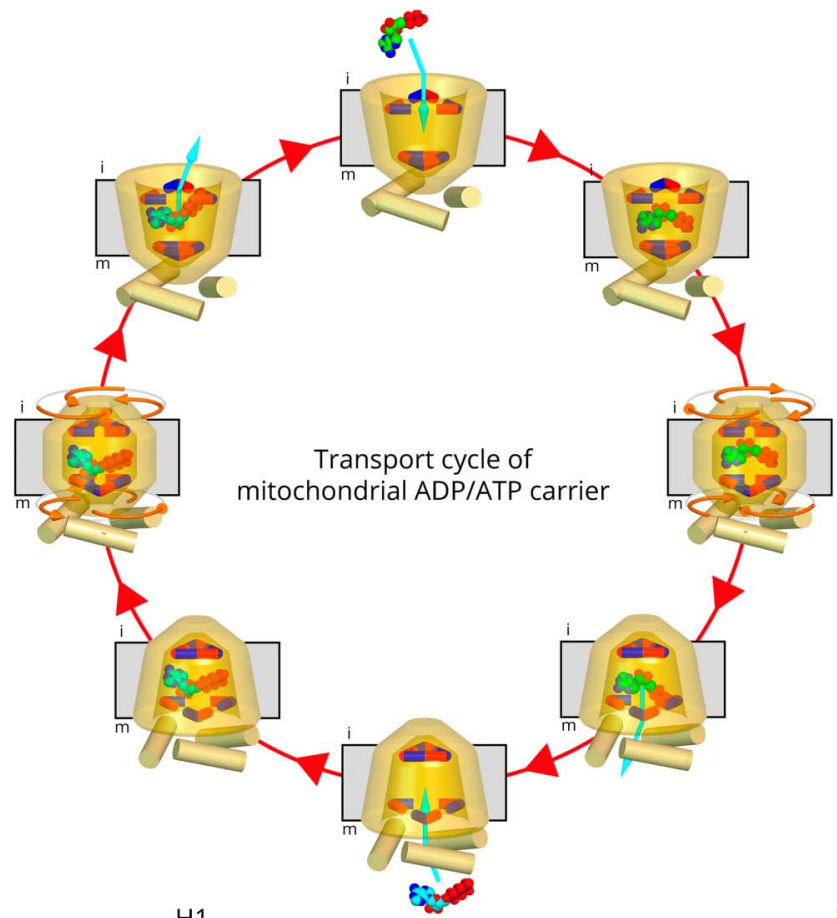

B

Homo sapiens

Mus musculus

Gallus gallus

Python bivittatus

Xenopus laevis

Danio rerio

Ciona intestinalis

Drosophila melanogaster

Caenorhabditis elegans

Saccharomyces cerevisiae
Arabidopsis thaliana

Arabidopsis that
Oryza sativa

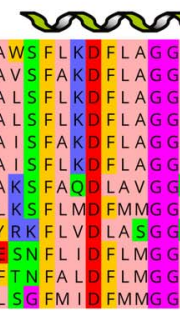

H1

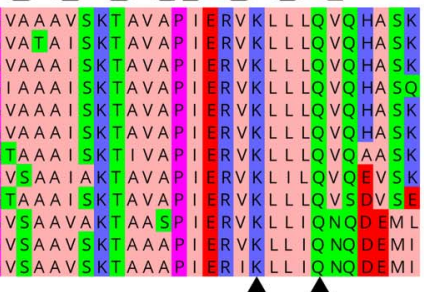

$\mathrm{H} 5$

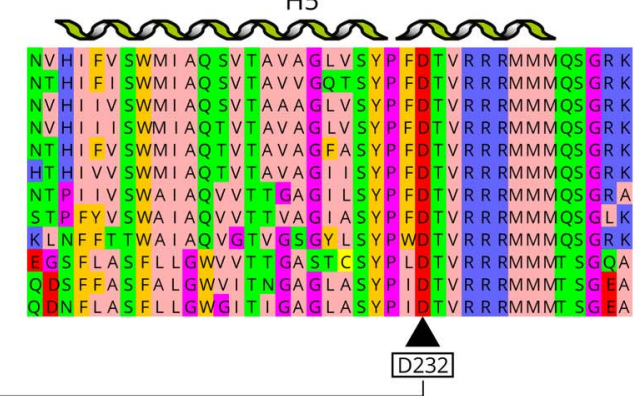

D232

C $\mathrm{OP}_{\mathrm{H} 5}$

D

K33 Q37
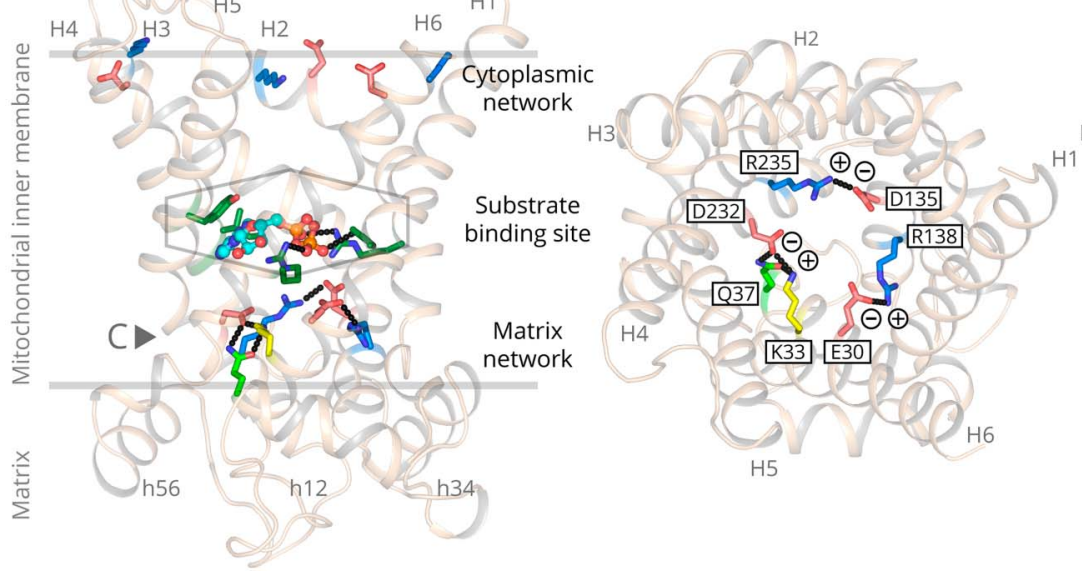

E

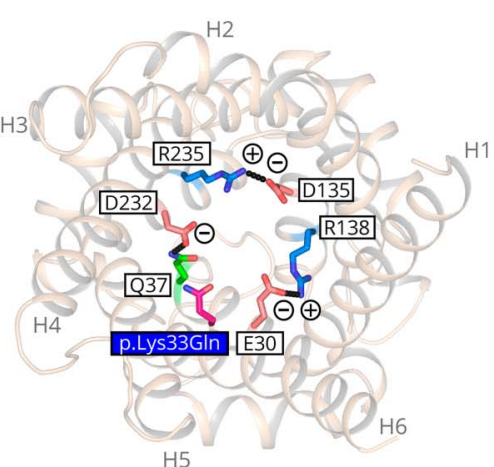

(A) Transport cycle of the mitochondrial AAC SLC25A4. The disruption and formation of salt bridges between positively (blue) and negatively (red) charged residues of the cytoplasmic and matrix networks, top and bottom respectively, change the access of the substrates to the central substrate binding site, indicated by a hexagon, from the intermembrane space (i) and matrix (m) side of the membrane. The imported ADP is shown in green sphere representation and the exported ATP in cyan. (B) Amino acid sequence alignment of AAC1 sequences of fungi, plants, and metazoan, showing that Lys33, Gln37, and Asp232 are highly conserved amino acid residues. (C) Lateral view of the human ADP/ATP carrier from the membrane, showing the residues of the matrix and cytoplasmic networks (blue and red sticks) and substrate binding site (green sticks, hexagon). ADP (light blue ball and stick) and the glutamine brace (light green stick) are also shown. Residue Lys33 that is mutated is shown in yellow. (D) Cytoplasmic view of the carrier showing only the residues of the matrix salt bridge network of SLC25A4 (blue and red sticks). (E) As (D), except for the p.Lys33GIn mutation, which is shown in magenta. The ionic interactions (black dash lines) are indicated with plus and minus signs. The model of human SLC25A4 was generated in SwissModel, using the structure of the closely related bovine mitochondrial AAC as template (PDB file: 1OKC). Adapted from Figure 3 in reference 4. AAC = ADP/ATP carrier; ADP = adenosine diphosphate; ATP = adenosine triphosphate. 

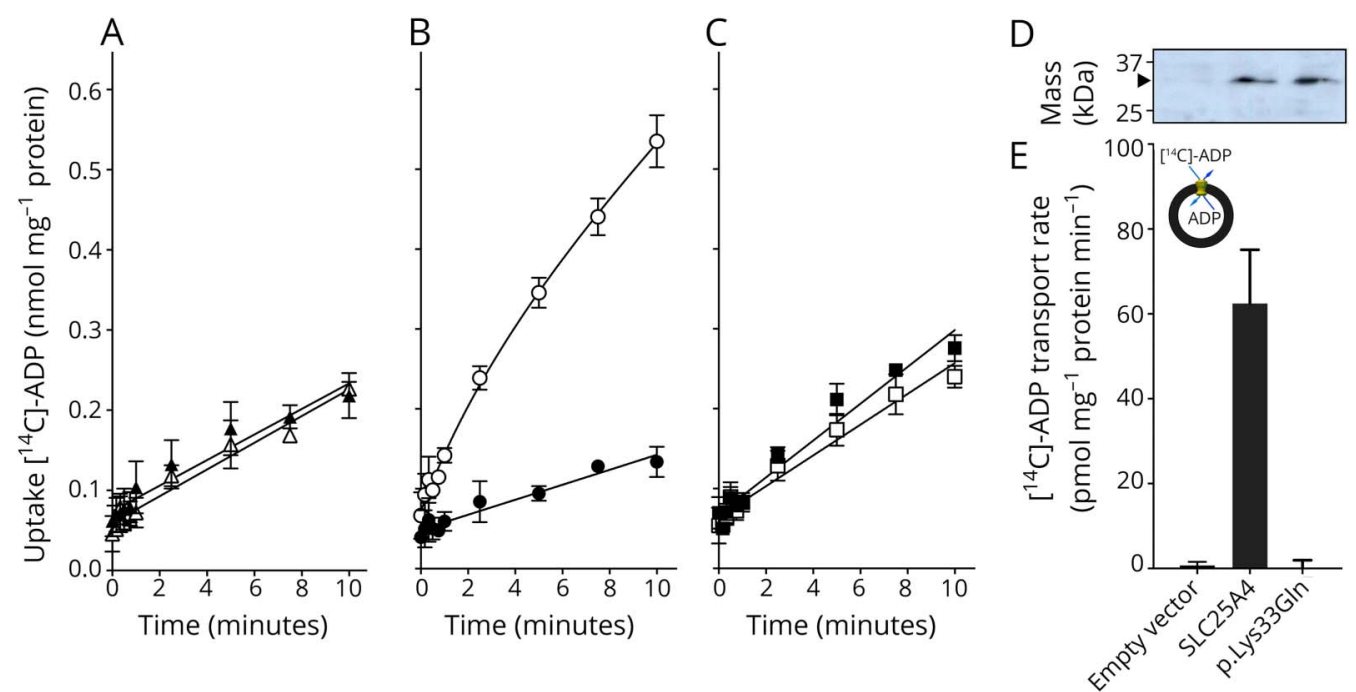

Transport of $\left[{ }^{14} \mathrm{C}\right]$-labeled ADP into vesicles of L. lactis membranes expressing (A) an empty vector control, (B) SLC25A4, or (C) SLC25A4 p.Lys33Gln in the presence (black symbols) or absence (white symbols) of $20 \mu \mathrm{M}$ carboxyatractyloside. Transport was initiated by the addition of $5 \mu \mathrm{M}\left[{ }^{14} \mathrm{C}\right]-\mathrm{ADP}$ and was terminated by filtration and washing at the indicated time intervals. The data are represented by the average and SD of 4 assays. (D) Expression levels of wild type and p.Lys33Gln determined by western blot analysis. (E) Transport rate of the empty vector control, human SLC25A4, and p.Lys33Gln, corrected for background binding. AAC = ADP/ATP carrier; ADP = adenosine diphosphate; ATP = adenosine triphosphate.

mutations associated with inherited PEO showing higher activity than mutations associated with de novo severe neonatal presentation. ${ }^{3}$

It remains unclear exactly why the presently documented case with the de novo dominant c.97A $>$ T(p.Lys33Gln) SLC25A4 variant presents with a much milder clinical phenotype than the other de novo cases ${ }^{3}$ despite the p.Lys33Gln mutant protein revealing essentially null ADP/ATP transport activity (figure $3 \mathrm{E}$ ). This case further supports that residual $\mathrm{ADP} /$ ATP transport activity of mutant AAC1 is only part of the puzzle. In vivo, there are 3 other $\mathrm{AAC}$ isoforms with varying expression levels in different tissues. Also, since the de novo variants are heterozygous, the relative expression ratios of wild-type vs mutant $\mathrm{AAC1}$, as well as other isoforms, will have an effect on the overall transport capacity. Their relative expression levels of carriers cannot be assessed from messenger RNA levels, as there are many poorly characterized steps involved in their biogenesis and turnover, and the available antibodies are unable to distinguish between the various isoforms.

Despite these issues, there is a clear correlation between the severity of OXPHOS dysfunction in skeletal muscle and the clinical phenotype between the current and previously reported de novo mutations. Patients with p.Arg80His or p.Arg235Gly mutations, showing severe neonatal presentations, had markedly decreased levels of total AAC $(<30 \%),{ }^{3}$ whereas there is only a slight decrease in the current patient (figure 1C). Similarly, the mosaic pattern of OXPHOS deficiency (figure 1, A and B) is not seen in previously documented de novo cases, where steady state protein levels of various OXPHOS components were completely undetectable. ${ }^{3}$ These data suggest that the clinical severity of de novo dominant mutations in SLC25A4 could be explained by the relative expression of wild-type and mutant alleles and the expression of other AAC isoforms, rather than the transport activity of the mutant variant alone.

This report expands the clinical phenotype for SLC25A4associated mitochondrial disease, with the mildest childhoodonset presentation to date.

\section{Author contributions}

X.R. Ortiz-Gonzalez, R.W. Taylor, and E.R.S. Kunji: study concept and design and study supervision. R.W. Taylor and E.R.S. Kunji: study funding. M.S. King, K. Thompson, S. Hopton, and L. He: experimental data acquisition and analysis. M.S. King and K. Thompson: statistical analysis for experimental data. X.R. Ortiz-Gonzalez: clinical data acquisition. X.R. Ortiz-Gonzalez, R.W. Taylor, E.R.S. Kunji, M.S. King, and K. Thompson: data interpretation and critical revision of the manuscript for important intellectual content.

\section{Study funding}

R.W. Taylor is supported by the Wellcome Centre for Mitochondrial Research (203105/Z/16/Z), the Medical Research Council (MRC) Centre for Translational Research in Neuromuscular Disease, Mitochondrial Disease Patient Cohort (UK) (G0800674), the Lily Foundation, the UK NIHR Biomedical Research Centre for Ageing and Age-related disease award to the Newcastle upon Tyne Foundation Hospitals NHS Trust, the MRC/EPSRC Molecular Pathology Node, and the UK NHS Highly Specialised Service for Rare Mitochondrial Disorders of Adults and Children. The research of M.S. King and 
E.R.S. Kunji was funded by the MRC programme grant MC UU_00015/1.X.R. Ortiz-Gonzalez is supported by the Robert Wood Johnson Foundation Harold Amos Faculty Development Award and the NIH (5K12NS049453-08).

\section{Disclosures}

K. Thompson, S. Hopton, L. He, E.R.S. Kunji, M.S. King, and R.W. Taylor report no disclosures. X.R. Ortiz-Gonzalez has received funding for travel/speaker honoraria from BioMarin and has received research support from the NINDS and the Robert Wood Johnson Foundation. Full disclosure form information provided by the authors is available with the full text of this article at Neurology.org/NG.

Received February 14, 2018. Accepted in final form May 15, 2018.

\section{References}

1. Kaukonen J, Juselius JK, Tiranti V, et al. Role of adenine nucleotide translocator 1 in mtDNA maintenance. Science 2000;289:782-785.
2. Palmieri L, Alberio S, Pisano I, et al. Complete loss-of-function of the heart/musclespecific adenine nucleotide translocator is associated with mitochondrial myopathy and cardiomyopathy. Hum Mol Genet 2005;14:3079-3088.

3. Strauss KA, Dubiner L, Simon M, et al. Severity of cardiomyopathy associated with adenine nucleotide translocator- 1 deficiency correlates with mtDNA haplogroup. Proc Natl Acad Sci USA 2013;110:3453-3458.

4. Thompson K, Majd H, Dallabona C, et al. Recurrent de novo dominant mutations in SLC25A4 cause severe early-onset mitochondrial disease and loss of mitochondrial DNA copy number. Am J Hum Genet 2016;99:860-876.

5. Kunji ER, Aleksandrova A, King MS, et al. The transport mechanism of the mitochondrial ADP/ATP carrier. Biochim Biophys Acta 2016;1863:2379-2393.

6. Dolce V, Scarcia P, Iacopetta D, Palmieri F. A fourth ADP/ATP carrier isoform in man: identification, bacterial expression, functional characterization and tissue distribution. FEBS Lett 2005;579:633-637.

7. Rocha MC, Grady JP, Grunewald A, et al. A novel immunofluorescent assay to investigate oxidative phosphorylation deficiency in mitochondrial myopathy: understanding mechanisms and improving diagnosis. Sci Rep 2015;5:15037.

8. Blakely E, He L, Gardner JL, et al. Novel mutations in the TK2 gene associated with fatal mitochondrial DNA depletion myopathy. Neuromuscul Disord 2008;18 557-560.

9. King MS, Boes C, Kunji ER. Membrane protein expression in Lactococcus lactis. Methods Enzymol 2015;556:77-97.

10. Ruprecht JJ, Hellawell AM, Harding M, Crichton PG, Mccoy AJ, Kunji ERS. Structures of yeast mitochondrial ADP/ATP carriers support a domain-based alternating-access transport mechanism. Proc Natl Acad Sci USA 2014;111: E426-E434. 


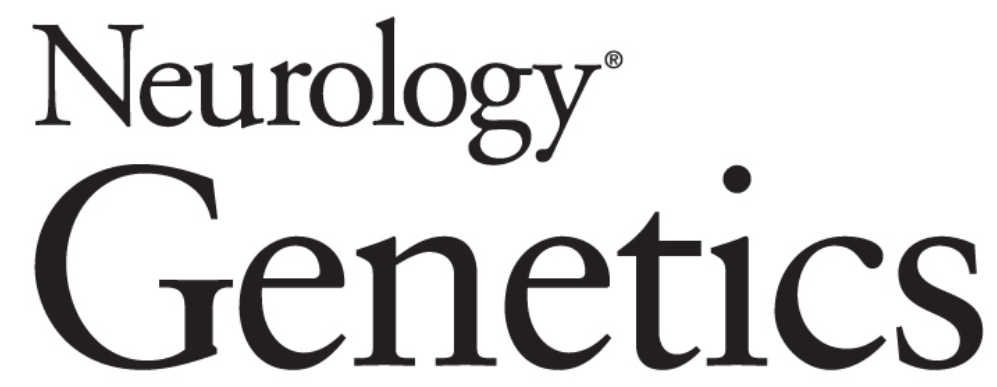

Expanding the phenotype of de novo SLC25A4-linked mitochondrial disease to include mild myopathy

Martin S. King, Kyle Thompson, Sila Hopton, et al. Neurol Genet 2018;4;

DOI 10.1212/NXG.0000000000000256

This information is current as of July 20, 2018

Neurol Genet is an official journal of the American Academy of Neurology. Published since April 2015, it is an open-access, online-only, continuous publication journal. Copyright Copyright ( 2018 The Author(s). Published by Wolters Kluwer Health, Inc. on behalf of the American Academy of Neurology.. All rights reserved. Online ISSN: 2376-7839.

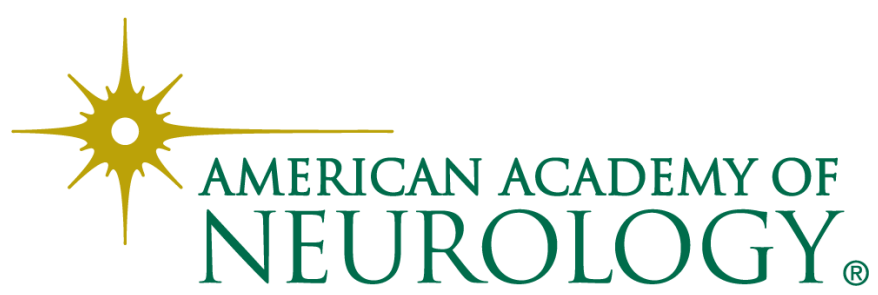




\section{Updated Information \& Services}

References

Citations

Subspecialty Collections

Permissions \& Licensing

Reprints including high resolution figures, can be found at: http://ng.neurology.org/content/4/4/e256.full.html

This article cites 10 articles, 3 of which you can access for free at: http://ng.neurology.org/content/4/4/e256.full.html\#\#ref-list-1

This article has been cited by 3 HighWire-hosted articles: http://ng.neurology.org/content/4/4/e256.full.html\#\#otherarticles

This article, along with others on similar topics, appears in the following collection(s):

\section{All Pediatric}

http://ng.neurology.org//cgi/collection/all_pediatric

Metabolic disease (inherited)

http://ng.neurology.org//cgi/collection/metabolic_disease_inherited Mitochondrial disorders

http://ng.neurology.org//cgi/collection/mitochondrial_disorders

Muscle disease

http://ng.neurology.org//cgi/collection/muscle_disease

Information about reproducing this article in parts (figures,tables) or in its entirety can be found online at:

http://ng.neurology.org/misc/about.xhtml\#permissions

Information about ordering reprints can be found online: http://ng.neurology.org/misc/addir.xhtml\#reprintsus

Neurol Genet is an official journal of the American Academy of Neurology. Published since April 2015, it is an open-access, online-only, continuous publication journal. Copyright Copyright ( 2018 The Author(s). Published by Wolters Kluwer Health, Inc. on behalf of the American Academy of Neurology.. All rights reserved. Online ISSN: 2376-7839.

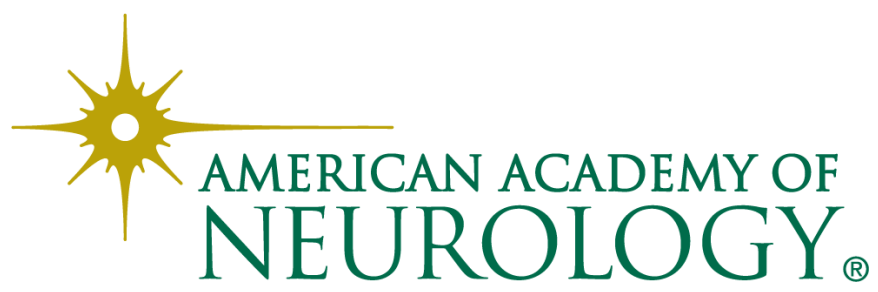

